\title{
On the Complexity of Clustering with Relaxed Size Constraints
}

\author{
Massimiliano Goldwurm ${ }^{(2)}$, Jianyi Lin ${ }^{(1)}$, Francesco Saccà ${ }^{(1)}$ \\ (1) Dipartimento di Informatica, (2) Dipartimento di Matematica \\ Università degli Studi di Milano, 20100 Milano - Italy
}

\begin{abstract}
We study the computational complexity of the problem of computing an optimal clustering $\left\{A_{1}, A_{2}, \ldots, A_{k}\right\}$ of a set of points assuming that every cluster size $\left|A_{i}\right|$ belongs to a given set $M$ of positive integers. We present a polynomial time algorithm for solving the problem in dimension 1 , i.e. when the points are simply rational values, for an arbitrary set $M$ of size constraints, which extends to the $\ell_{1}$-norm an analogous procedure known for the $\ell_{2}$-norm. Moreover, we prove that in the Euclidean plane, i.e. assuming dimension 2 and $\ell_{2}$-norm, the problem is NP-hard even with size constraints set reduced to $M=\{2,3\}$.
\end{abstract}

Keywords: geometric clustering problems; cluster size constraints; computational complexity; constrained k-Means

\section{Introduction}

In the area of unsupervised machine learning and statistical data analysis the clustering methods play an important role with applications in pattern recognition, bioinformatics, signal and image processing, medical diagnostics. Clustering consists in grouping a set of objects into subsets, called clusters, that are maximally homogeneous [5]8. Partitional or hard clustering requires the subsets to be disjoint and non-empty, and in the usual geometric setting the similarity between objects is measured by distance between points representing the objects [15.

A classical clustering problem is the so-called Euclidean Minimum-Sum-ofSquares [1, Variance-based [10] or $k$-Means clustering problem: given a finite point set $X \subset \mathbb{R}^{d}$, find a $k$-partition $\left\{A_{1}, \ldots, A_{k}\right\}$ of $X$ minimizing the sum of weights $W\left(A_{1}, \ldots, A_{k}\right)=\sum_{i} W\left(A_{i}\right)=\sum_{i} \sum_{x \in A_{i}}\left\|x-\mu\left(A_{i}\right)\right\|^{2}$ of all clusters, where $\mu\left(A_{i}\right)$ is the sample mean of $A_{i}$ and $\|\cdot\|$ is the Euclidean norm. This partitional clustering problem is difficult: when $d$ is part of the instance the problem is NP-hard even if the number of clusters is fixed to $k=2$ [1]; the same occurs for arbitrary $k$ with fixed dimension $d=2$ [7/16]. Nonetheless, a wellknown heuristic for this problem is Lloyd's algorithm [14, also named $k$-Means Algorithm, which is not guaranteed to converge to the global optimum. This algorithm is usually very fast, but may require exponential time in the worst case [22]. 
Often one has some a-priori information on the clusters, that can be incorporated into traditional clustering techniques to increase the clustering performance [2. Problems that include background information are so-called constrained clustering and can be divided into two classes based on the constraints: instance-level constraints typically define pairs of elements that must be (mustlink) or cannot be (cannot-link) in the same cluster 25, and cluster-level constraints prescribe characteristics of each cluster, such as cluster diameter or cluster size [6|21]. In [26] cluster size constraints are used for improving clustering accuracy, for instance allowing one to avoid extremely small or large clusters in standard cluster analysis. In the size constrained clustering (SCC) problem, assuming an $\ell_{p}$-norm (we suppose $p \in \mathbb{N}_{+}$throughout this work), typically one is given a finite set $X \subset \mathbb{R}^{d}$ of $n$ points and $k$ positive integers $m_{1}, \ldots, m_{k}$ such that $\sum_{i} m_{i}=n$, and searches for a partition $\left\{A_{1}, \ldots, A_{k}\right\}$ of $X$, with $\left|A_{1}\right|=m_{1}, \ldots,\left|A_{k}\right|=m_{k}$, that minimizes the objective function $W\left(A_{1}, \ldots, A_{k}\right)=$ $\sum_{i=1}^{k} \sum_{x \in A_{i}}\left\|x-c_{i}\right\|_{p}^{p}$, where each $c_{i}=\operatorname{argmin}_{c \in \mathbb{R}^{d}} \sum_{x \in A_{i}}\|x-c\|_{p}^{p}$ is the $\ell_{p^{-}}$ centroid of $A_{i}$.

For arbitrary $k \in \mathbb{N}$, this problem is NP-hard also in dimension $d=1$, for any (fixed) $\ell_{p}$-norm, $p \geq 1$; the same negative result holds for arbitrary $d \in \mathbb{N}$ when the number of clusters is fixed to $k=2$, for every $\ell_{p}$-norm with $p>1[3$. On the contrary, in the case $d=2=k$ the problem is solvable in $O\left(n^{2} \log n\right)$ time assuming Manhattan norm $\left(\ell_{1}\right)$ and in $O\left(n \sqrt[3]{m} \log ^{2} n\right)$ time with Euclidean norm $\left(\ell_{2}\right)$ [13, where $m$ is the size of one of the two clusters.

In this work we study a relaxed version of the size constrained clustering problem, where the size of each cluster belongs to given set $M$ of integers. We show that in dimension $d=1$, for an arbitrary (finite) $M \subset \mathbb{N}$, assuming the Manhattan norm, the solution can be obtained in $O(n(k s+n))$ time, where $k$ is the number of clusters and $s$ is the cardinality of $M$. This extends an analogous algorithm [4 proposed for the Euclidean norm and applied to computational biology problems as a method for identification of promoter regions in genomic sequences. Note instead that, in dimension 1, the SCC problem is NP-hard [3]. On the contrary, in dimension $d=2$, we prove that even fixing $M=\{2,3\}$ the problem is NP-hard with Euclidean norm.

\section{Problem definition}

In this section we define the problem and fix our notation. Given a positive integer $d$, for every real $p \geq 1$ and every point $a=\left(a_{1}, \ldots, a_{d}\right) \in \mathbb{R}^{d}$, we denote by $\|a\|_{p}$ the $\ell_{p}$-norm of $a$, i.e. $\|a\|_{p}=\left(\sum_{1}^{d}\left|a_{i}\right|^{p}\right)^{1 / p}$. Clearly, $\|a\|_{2}$ and $\|a\|_{1}$ are the Euclidean and the Manhattan (or Taxicab) norm of $a$, respectively.

Given a finite set $X \subset \mathbb{R}^{d}$, a cluster of $X$ is a non-empty subset $A \subset X$, while a clustering is a partition $\left\{A_{1}, \ldots, A_{k}\right\}$ of $X$ in $k$ clusters for some $k$. Assuming the $\ell_{p}$ norm, the centroid and the weight of a cluster $A$ are the values $C_{A} \in \mathbb{R}^{d}$ and $W_{p}(A) \in \mathbb{R}_{+}$defined, respectively, by

$$
C_{A}=\underset{c \in \mathbb{R}^{d}}{\operatorname{argmin}} \sum_{a \in A}\|a-c\|_{p}^{p}, \quad W_{p}(A)=\sum_{a \in A}\left\|a-C_{A}\right\|_{p}^{p}
$$


The weight of a clustering $\left\{A_{1}, \ldots, A_{k}\right\}$ is $W_{p}\left(A_{1}, \ldots, A_{k}\right)=\sum_{1}^{k} W_{p}\left(A_{i}\right)$. We recall that, in case of $\ell_{2}$-norm, the weight of a cluster $A$ can be computed by relation

$$
W_{2}(A)=\frac{1}{|A|} \sum_{(*)}\|a-b\|_{2}^{2}
$$

where the sum is extended to all unordered pairs $\{a, b\}$ of distinct elements in $A$. Moreover, given a set $\mathcal{M} \subset \mathbb{N}$, any clustering $\left\{A_{1}, \ldots, A_{k}\right\}$ such that $\left|A_{i}\right| \in \mathcal{M}$ for every $i=1, \ldots, k$, is called $\mathcal{M}$-clustering.

RSC- $d$ Problem (with $\ell_{p}$-norm): Relaxed Size Constrained Clustering in $\mathbb{R}^{d}$ Given a set $X \subset \mathbb{Q}^{d}$ of $n$ points, an integer $k$ such that $1<k<n$ and a finite set $\mathcal{M}$ of positive integers, find an $\mathcal{M}$-clustering $\left\{A_{1}, \ldots, A_{k}\right\}$ of $X$ that minimizes $W_{p}\left(A_{1}, \ldots, A_{k}\right)$.1

When $\mathcal{M}$ is not included in the instance, but fixed in advance, we call the problem $\mathcal{M}$-RSC- $d$ (with $\ell_{p}$-norm). In this work we study these problems in dimension $d=1,2$ assuming $\ell_{1}$ and $\ell_{2}$-norm.

\section{Dynamic programming for RSC on the line}

In this section we describe a polynomial-time algorithm for RSC-1 that works assuming either $\ell_{1}$ or $\ell_{2}$-norm. This procedure is based on a dynamic programming technique, in the style of [19, based on the so-called String Property [24]3. A simplified version of the procedure in the case of $\ell_{2}$-norm is also presented in [4] and applied to problems of computational biology.

Consider an instance $(X, k, \mathcal{M})$ of RSC-1, where $X=\left(x_{1}, x_{2} \ldots, x_{n}\right)$ is a sorted sequence of rational numbers, $k \in\{1, \ldots, n-1\}$ and $|\mathcal{M}|=s \leq n$. For any $1 \leq i \leq j \leq n$, let $X[i, j]$ be the subsequence $\left(x_{i}, x_{i+1}, \ldots, x_{j}\right)$. For a given $p \in\{1,2\}$, we define the $n \times n$ matrix $U=[U(i, j)]_{i, j=1, \ldots, n}$ by setting $U(i, j)=W_{p}(X[i, j])=\sum_{t=i}^{j}\left|x_{t}-C_{X[i, j]}\right|^{p}$ if $j-i+1 \in \mathcal{M}$ and $U(i, j)=\infty$ otherwise, that is the weight of cluster $X[i, j]$ when it has admissible size.

Lemma 1. Given $p \in\{1,2\}$, for every instance $(X, k, \mathcal{M})$ of $R S C-1$ with $|X|=$ $n$, matrix $U$ can be computed in $O\left(n^{2}\right)$ time.

Proof. First, assume $p=2$. In this case it is easy to check that the weight of any cluster $A$ is $W_{2}(A)=\sum_{a \in A} a^{2}-\frac{1}{|A|}\left(\sum_{a \in A} a\right)^{2}$. Denoting $Q(i):=\sum_{j=1}^{i} x_{j}^{2}$ and $S(i):=\sum_{j=1}^{i} x_{j}$, the finite entries of matrix $U$ reduce to

$$
U(i, j)=Q(j)-Q(i-1)-\frac{1}{j-i+1}(S(j)-S(i-1))^{2} .
$$

The sequences $Q$ and $S$ can be computed in linear time, and thus the computation of (2) requires constant time for each $i, j$. Hence, matrix $U$ can be computed in $O\left(n^{2}\right)$ time in case $p=2$.

\footnotetext{
${ }^{1}$ If $X$ does not admit a $\mathcal{M}$-clustering then symbol $\perp$ is returned.
} 
When $p=1$, the weight $W_{1}(X[i, j])$ is the sum of the distances between elements and median of $X[i, j]$. Denote $m:=(i+j) / 2$ and for any cluster $X[i, j]$ set the left and right sums $L(i, j):=\sum_{i<h<m} x_{h}$ and $R(i, j):=\sum_{m<h \leq j} x_{h}$. It can be checked (3, Prop. 10]) that $W_{1}(\bar{X}[i, j])=R(i, j)-L(i, j)$. Since $X[i, j]$ is sorted, it can be seen that, for $i<j$,

$$
\begin{aligned}
& L(i, j)=L(i, j-1) \text { if } m \in \mathbb{N}, \quad L(i, j)=L(i, j-1)+x_{\lfloor m\rfloor} \text { otherwise, } \\
& R(i, j)=R(i, j-1)-x_{m}+x_{j} \text { if } m \in \mathbb{N}, \quad V=R(i, j-1)+x_{j} \text { otherwise }
\end{aligned}
$$

and $L(i, i)=R(i, i)=0$. By means of these recursive formulae the quantities $L(i, j), R(i, j), W_{1}(X[i, j])$, for all $i \leq j$, can be computed in $O\left(n^{2}\right)$ time, and hence the same holds for determining matrix $U$ when $p=1$.

Now, for every $h \in\{1, \ldots, k\}$ and every $j \in\{1, \ldots, n\}$, let $Z(h, j)$ be the weight of a solution of RSC-1 for the instance $(X[1, j], h, \mathcal{M})$ in case $h \leq j$, while $Z(h, j)=\infty$ if $h>j$. These values can be derived from $U$.

Proposition 2. The following properties hold:

i) $Z(1, j)=U(1, j)$ for all $j=1, \ldots, n$;

ii) $Z(h, j)=\min _{m \in \mathcal{M}}(Z(h-1, j-m)+U(j-m+1, j))$ for all $h=2, . ., k ; j=1, \ldots, n$.

Proof. Case $i)$ is obvious. Since $Z(h, j)$ is the weight of the optimal solution for $(X[1, j], h, \mathcal{M})$, the corresponding solution $\left\{A_{1}, \ldots, A_{h}\right\}$ satisfies the String Property, i.e. each cluster $A_{i}$ consists of consecutive points of $X$ 243].

Then, its right-most cluster $A_{h}$ has size $\left|A_{h}\right|=m \in \mathcal{M}$ and weight $W_{p}\left(A_{h}\right)=$ $W_{p}(X[j-m+1, j])=U(j-m+1, j)$.

The other clusters $A_{1}, \ldots, A_{h-1}$ form a feasible clustering of RSC-1 for the instance $(X[1, j-m], h-1, \mathcal{M})$, which has minimum weight $W_{p}\left(A_{1}, \ldots, A_{h-1}\right)=$ $\sum_{1}^{h-1} W_{p}\left(A_{i}\right)=Z(h-1, j-m)$, otherwise it is easy to check that also $\left\{A_{1}, \ldots, A_{h}\right\}$ would not be an optimal solution for $(X[1, j], h, \mathcal{M})$.

As a consequence, $Z(h, j)=Z(h-1, j-m)+U(j-m+1, j)$ for some $m \in \mathcal{M}$, and since $Z(h, j)$ has to take the minimum value, property $i i)$ is proved.

Relying on the previous proposition we can design an algorithm for RSC-1.

Theorem 3. For any $p \in\{1,2\}, R S C-1$ with $\ell_{p}$-norm can be solved in $O(n(k s+$ $n)$ ) time and $O\left(n^{2}\right)$ space.

Proof. By Lemma 1 we first compute matrix $U$ in $O\left(n^{2}\right)$ time. Then, by means of Proposition 2, matrix $Z=[Z(h, j)]_{h=1, \ldots, k ; j=1, \ldots, n}$ can be computed row by row. Each entry requires at most $s=|\mathcal{M}|$ sums and comparisons. The computation is described by the following scheme, where we store in $\ell_{h, j}$ the size of the last cluster of the optimal solution for $(X[1, j], h, \mathcal{M})$, for each pair of indices $h, j$.

begin

$Z:=\{\infty\}^{k \times n}$ 


$$
\begin{aligned}
& \text { for } j=1, \ldots, n \text { do }\left\{\begin{array}{l}
Z(1, j):=U(1, j) \\
\text { if } U(1, j) \neq \infty \text { then } \ell_{1, j}:=j
\end{array}\right. \\
& \text { for } h=2, \ldots, k \text { do } \\
& \text { for } j=h, \ldots, n \text { do } \\
& \hat{m}:=\operatorname{argmin}_{m \in \mathcal{M}}\{Z(h-1, j-m)+U(j-m+1, j)\} \\
& \text { if } \hat{m} \text { is well-defined then } \\
& Z(h, j):=Z(h-1, j-\hat{m})+U(j-\hat{m}+1, j) \\
& \ell_{h, j}:=\hat{m}
\end{aligned}
$$

Clearly, if $\ell_{k, n}$ is not defined then the symbol $\perp$ is returned since no admissible clustering for $(X, k, \mathcal{M})$ exists. Otherwise, the solution of the problem can be obtained by the following procedure:

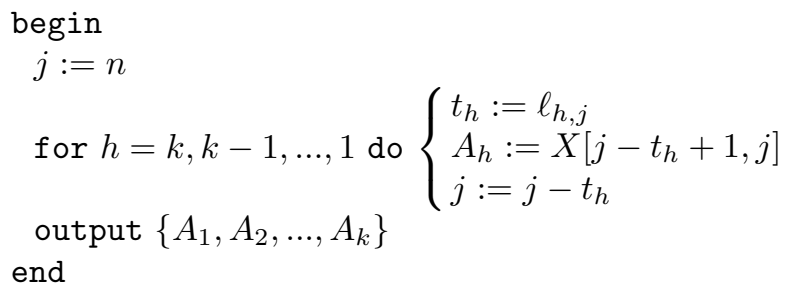

The overall time required to compute matrices $U$ and $Z$ is $O(n(k s+n))$. The space necessary to maintain all tables is $O\left(n^{2}\right)$ since $k<n$.

It is worth noting that the analogous problem, where the size of each cluster is fixed by the instance, is NP-hard even in dimension $d=1$ for every $\ell_{p}$-norm 3. This shows that the form of the size constraints for clustering problems is relevant for the existence of polynomial time algorithms.

\section{NP-hardness of RSC in the Euclidean Plane}

In this section we show that, assuming $\ell_{2}$-norm, the $\{2,3\}$-RSC-2 problem is NPhard, and therefore RSC-2 also is NP-hard. To this end we introduce a decision version of the problem and describe a polynomial-time reduction from Planar 3-SAT.

Decision $\{2,3\}$-RSC-2 Problem

Given a point set $X=\left\{p_{1}, \ldots, p_{n}\right\} \subset \mathbb{Q}^{2}$, an integer $k, 1<k \leq n / 2$, and a rational value $\lambda>0$ (threshold), decide whether there exists a $\{2,3\}$-clustering $\left\{A_{1}, \ldots, A_{k}\right\}$ of $X$, consisting of $k$ clusters, such that $W_{2}\left(A_{1}, \ldots, A_{k}\right) \leq \lambda$.

Recall that a 3 -CNF formula $\Phi$ is a boolean formula given by the conjunction of clauses each of which has 3 literals. If $V$ and $C$ are, respectively, the set of variables and the set of clauses of $\Phi$, the graph of $\Phi$ is defined as the undirected bipartite graph $G_{\Phi}$ such that $V \cup C$ is the family of nodes and $E=\{\{v, c\}: v \in$ $V, c \in C$, and either $v$ or $\bar{v}$ appears in $c\}$ is the set of edges. A formula $\Phi$ is said 
to be planar if $G_{\Phi}$ is planar. The Planar 3-SAT problem consists in deciding whether a planar 3-CNF formula $\Phi$ is satisfiable.

It is known that Planar 3-SAT is strongly NP-complete [12. It is also proved that it suffices to consider formulae whose associated graph can be embedded in $\mathbb{R}^{2}$, with variables arranged on a straight line, and with clauses arranged above and below the straight line [11. Moreover, the edges between variables and clauses can be drawn in a rectilinear fashion [17.

We also recall that a box-orthogonal drawing of a graph $G$ is a planar embedding of $G$ on an integer grid where each vertex is mapped into a (possibly degenerate) rectangle and each edge becomes a path of horizontal or vertical segments of the grid. Rectangles are disjoint and paths do not intersect. Any planar graph of $n$ nodes admits a box-orthogonal drawing computable in $O(n)$ time that uses a $a \times b$ grid, where $a+b \leq 2 n$ [9, Th. 3].

Our goal is to show that Planar 3-SAT is reducible in polynomial time to Decision $\{2,3\}$-RSC-2. The proof is obtained by adapting the reduction from Planar 3-SAT to an unconstrained version of the $k$-means problem in the plane, presented in [16. Here, the main difference is that in our construction we determine directly the rational coordinates of the points given by the reduction, avoiding the approximation of irrational values. Moreover, our reduction does not yield multiple copies of the same point in the plane.

To describe the reduction we show how an arbitrary planar 3-CNF formula $\Phi$, can be associated with an instance $(X, k, \lambda)$ of the Decision $\{2,3\}$-RSC-2, computable in polynomial time w.r.t. $|\Phi|$, such that $\Phi$ is satisfiable if and only if $X$ admits a partition into $k$ clusters of cardinality 2 or 3 , having a total weight at most $\lambda$. The definition of such a reduction is split in several phases: the first one computes an embedding of graph $G_{\Phi}$ into a planar integer grid; the others determine the rational coordinates of points in $X$, and the values $k$ and $\lambda$.

The general idea is to build an embedding of $G_{\Phi}$ by representing each clause by a point in the grid, and associating each variable with a cycle on the grid that connects all points of clauses containing the variable. Clearly, these cycles do not overlap, and each clause-point is touched exactly by 3 cycles. Now, the points of $X$ are placed along every cycle, so that there are only 2 optimal $\{2\}$ clusterings for the points of each cycle, which may be associated to the truth assignments of the variable. The satisfiability of each clause will correspond to the possibility of clustering the clause-point with the nearest pair in one of the optimal $\{2\}$-clusterings associated to a variable occurring in the clause.

\section{1) Embedding of $G_{\Phi}$ into a planar grid}

The first phase is described by the following steps, illustrated in Figure 1 . Step 0. Compute the box-orthogonal drawing $D$ of $G_{\Phi}$ as stated above. We can map any variable into a (non-empty) rectangle and any clause into a vertex of the grid. Moreover, the base of all rectangles can be put on the same horizontal straight line, and the vertices representing clauses above or below such a line.

Step 1. Expand the previous drawing by a factor of 2 and call $D_{1}$ the new drawing. This doubles all distances between vertices in $D$. 
Step 2. Shift $D_{1}$ half unit upward and rightward and let $D_{2}$ be the new drawing. Now, each clause corresponds to a point in the centre of a unit square of the grid, and each path from a rectangle (variable) to a point (clause) crosses just in the middle some unit sides of the grid.

Step 3. Expand all rectangles by half grid unit in all four vertical and horizontal directions, and replace any point (clause) of $D_{2}$ by a unit square centred at the same location, erasing the overlapping portion (half unit long) of paths. We call $D_{3}$ the new drawing. Now, all rectangles have sides of odd length and no path in $D_{3}$ starts from a vertex of a rectangle.

Step 4. Replace every path from a rectangle (variable) to a unit square (clause) by a strip of unit width on the grid that cover the same path, erasing the boundary portion of rectangle overlapping the strip. The resulting drawing is called $D_{4}$. Now every variable $v$ corresponds to a (sort of) cycle on the grid that includes both the residual rectangle representing $v$ and all strips towards the unit squares (clauses) where $v$ occurs, together with one side for each touched square.

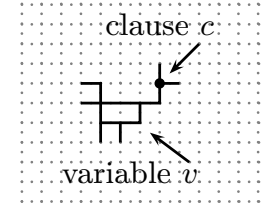

$D$

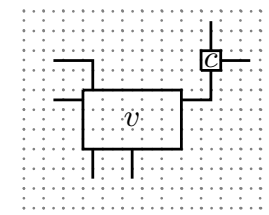

$D_{3}$

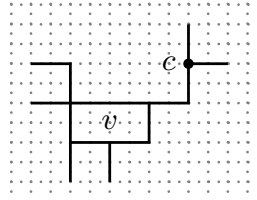

$D_{1}$

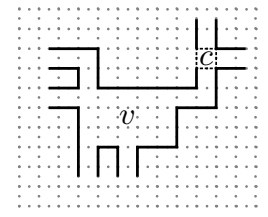

$D_{4}$

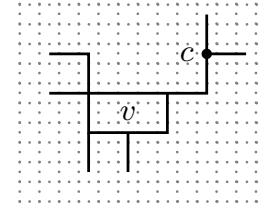

$D_{2}$

Expand drawing $D_{4}$ by a factor of

15

$D_{5}$

Fig. 1. Main steps of the graph transformations used in the reduction.

Step 5. Expand the previous drawing by a factor of 15 . We call $D_{5}$ the new drawing. Thus, each clause is now associated with a square on the grid having side of length 15, while the strips described in Step 4 are formed by parallel segments at distance 15 to each other. Moreover, in the following we call borders the straight-line segments forming the cycles associated with the variables.

\section{2) Definition of point set $X$}

Let $V=\left\{v_{1}, \ldots, v_{n}\right\}$ and $C=\left\{c_{1}, \ldots, c_{m}\right\}$ be, respectively, the set of variables and the set of clauses of $\Phi$. First, for every $c_{j} \in C, X$ contains a point $z_{j} \in \mathbb{Q}^{2}$ located near the centre of the square associated with $c_{j}$. The exact position of each $z_{j}$ is defined by Fig. 2. where the cycles are represented by dashed lines and the sides of the square are removed for sake of simplicity.

Moreover, for every variable $v_{i} \in V, X$ contains a circuit $\Gamma_{i}$ of $2 L_{i}$ consecutive points $\left\{x_{i 1}, x_{i 2}, \ldots, x_{i\left(2 L_{i}\right)}\right\}$, for a suitable integer $L_{i}$. With few exceptions (as in Fig. (2), all $x_{i \ell}$ 's lie inside the cycle of drawing $D_{5}$ associated with $v_{i}$ and inside 
the square associated with the clauses where $v_{i}$ occurs. The idea is to put almost all points at distance 2 from the borders, setting at distance 5 from each other most consecutive points $x_{i t}, x_{i(t+1)}$, as well as points $x_{i\left(2 L_{i}\right)}$ and $x_{i 1}$. Hence

$$
X=\left\{z_{j} \mid j=1,2, \ldots, m\right\} \cup\left\{x_{i \ell} \mid i=1,2, \ldots, n, \ell=1,2, \ldots, 2 L_{i}\right\}
$$

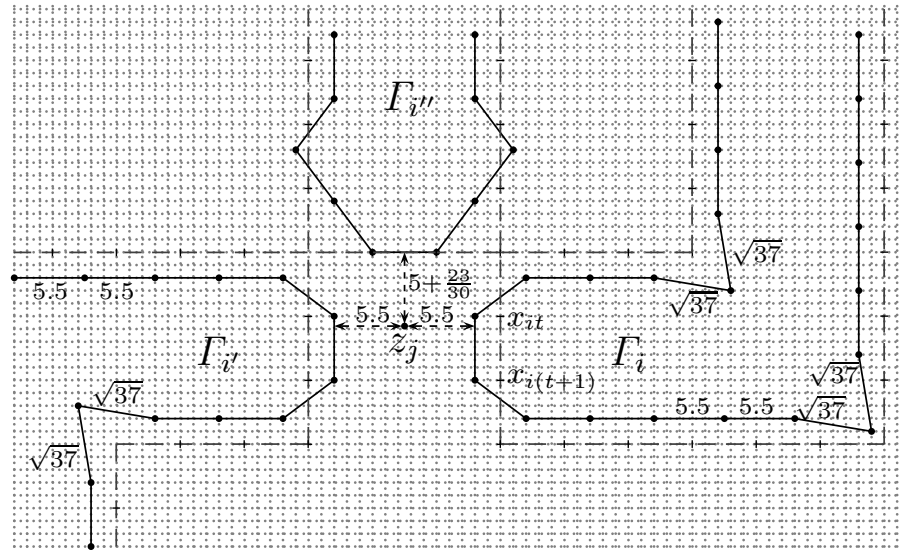

Fig. 2. Points of 3 circuits in the neighbourhood of a clause-point $z_{j}$. Edges with length different from 5 are indicated.

The precise position of points $x_{i \ell}$ 's is illustrated in Figs. 2 and 3 and is formally defined by conditions a), b), c) given below. Such a position depends on the angles, inside the cycle associated with $v_{i}$, formed between two incident borders. Every angle has measure either $\pi / 2$ or $3 \pi / 2$; in the first case we say the angle is convex, in the second case we say it is concave (e.g., in Fig. 3. angle $\beta$ is convex, while $\alpha$ is concave).

a) Near every convex (resp. concave) angle three consecutive points of $\Gamma_{i}$ are placed as shown by angles $\beta, \delta, \varepsilon, \zeta, \eta$ (resp. $\alpha, \gamma, \theta, \iota$ ) in Fig. 3. Note that the second point of the triple always lies on the bisector.

b) Between any two consecutive angles, the other points of $\Gamma_{i}$ are put on a straight-line at distance 2 from the border, so that consecutive points are set at distance 5 from each other, with the exception of two segments of length 4.5 (respectively, 5.5) if both angles are concave (resp., convex). As examples, see in Fig. 3 points between angles $\beta$ and $\gamma, \iota$ and $\theta, \delta$ and $\varepsilon$.

c) If $v_{i}, v_{i^{\prime}}, v_{i^{\prime \prime}}$ are the variables occurring in a clause $c_{j}$, then near the square of size $15 \times 15$ associated with $c_{j}$, points of $\Gamma_{i}, \Gamma_{i^{\prime}}, \Gamma_{i^{\prime \prime}}$ are set as defined in Fig. 2. Note that here, all pair of consecutive points are at distance 5 from each other with two exceptions:

- triple of points close to angles are located according to condition a);

- before convex angles, points are located to form two consecutive segments of length 5.5 . 


\section{3) Weight of clusters}

Note that all pairs of consecutive points in any $\Gamma_{i}$ form a segment having one of the following lengths: $4.5,5,5.5, \sqrt{37}$. The weight of the corresponding clusters is easily obtained from Eq. (1): 10.125, 12.5, 15.125, 18.5.

Moreover, every set $\Gamma_{i}$ admits only two $\{2\}$-clusterings of minimum weight, consisting of pairs of consecutive points, given by

$$
\begin{gathered}
\pi_{1}(i)=\left\{\left\{x_{i u}, x_{i(u+1)}\right\} \mid u=1,3,5, \ldots, 2 L_{i}-1\right\} \quad \text { and } \\
\pi_{2}(i)=\left\{\left\{x_{i u}, x_{i(u+1)}\right\} \mid u=2,4,6, \ldots, 2 L_{i}-2\right\} \cup\left\{\left\{x_{i\left(2 L_{i}\right)}, x_{i 1}\right\}\right\}
\end{gathered}
$$

For simplicity, hereafter we call segment (respectively, triangle) a cluster of cardinality 2 (resp., 3 ).

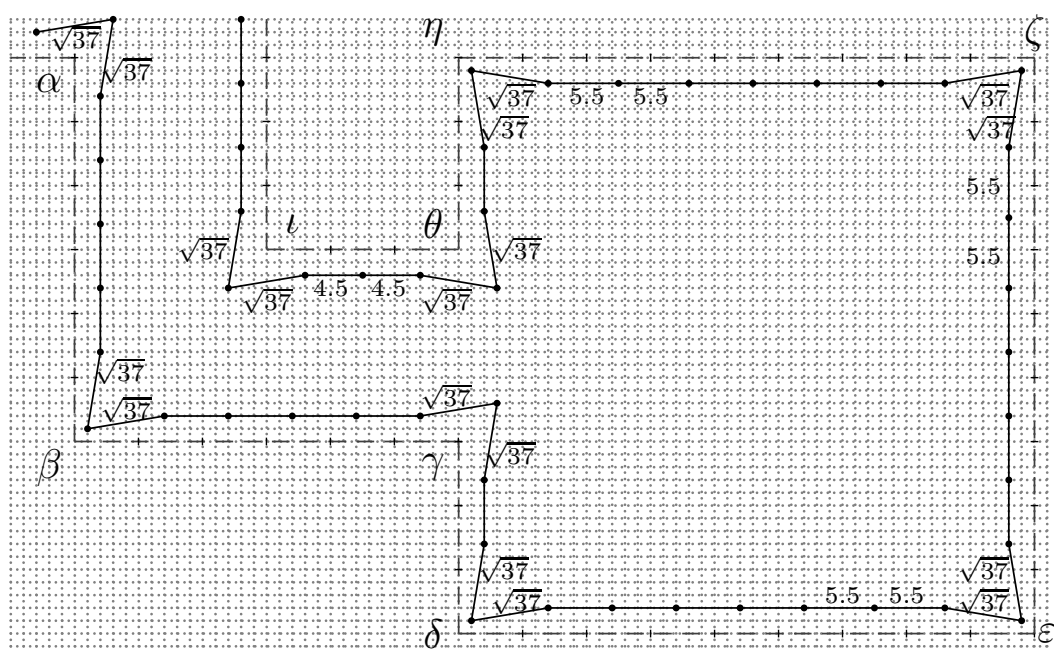

Fig. 3. Points of a circuit $\Gamma_{i}$ inside the corresponding rectangle. Segments with length different from 5 are indicated. Note that angles $\alpha, \gamma, \theta, \iota$ are concave, while $\beta, \delta, \varepsilon, \zeta, \eta$ are convex.

Now, consider a clause $c_{j}$ containing a variable $v_{i}$ (as a positive or negative literal) and let $x_{i t}, x_{i(t+1)}$ be the pair of points of $\Gamma_{i}$ nearest to point $z_{j}$. We say that $z_{j}$ touches the segment $\left\{x_{i t}, x_{i(t+1)}\right\}$. Clearly every $z_{j}$ touches three segments, one for each variable appearing in $c_{j}$. Note from Fig. 2, that the distance between $z_{j}$ and a touched segment is either 5.5 or $5+\frac{23}{30}$. Then, using Eq. (1), by elementary computation we can determine the weight of any triangle formed by each $z_{j}$ with its touched segments, as well as the weight of every triangle of consecutive points in any $\Gamma_{i}$. Such a direct computation proves the following property.

Lemma 4. If point $z_{j}$ touches a segment $\left\{x_{i t}, x_{i(t+1)}\right\}$ then the weight of triangle $\left\{z_{j}, x_{i t}, x_{i(t+1)}\right\}$ is given by $w=\frac{23402}{675}$, which satisfies $34.66<w<34.67$. Moreover, every triangle composed by points of $\Gamma_{i}$ has weight greater than $w$. 


\section{4) Parity condition}

By a suitable choice of the first point $x_{i 1}$, and possibly by adding new points to $\Gamma_{i}$ (as explained below), we can assume that the following parity condition holds: in any $\Gamma_{i}$, every segment touched by a point $z_{j}$ belongs to either $\pi_{1}(i)$ or $\pi_{2}(i)$ according to whether $v_{i}$ or $\overline{v_{i}}$ appears in $c_{j}$, respectively. In order to guarantee this property, slight changes to points of $\Gamma_{i}$ near the square including $z_{j}$ may be necessary, which are illustrated in Figure 4. This change add two new points (one before and one after the touched segment), and determines 4 more segments of length 4.5, two of which are to be included into $\pi_{1}(i)$, the others into $\pi_{2}(i)$. In order to apply this transformation the circuit must contain a rectilinear portion of length at least 30, either horizontal or vertical, as shown in Fig. 4 (left). We may always assume this is satisfied by requiring one more expansion of the initial drawing by a factor of 2 (executing Step 1 twice in the embedding phase).
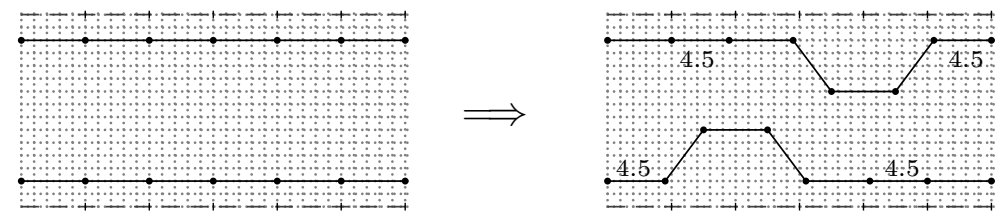

Fig. 4. (Left) $30 \times 15$ horizontal strip preserving parity. (Right) $30 \times 15$ horizontal strip for changing parity. The vertical case in analogous.

\section{5) Definition of $k$ and $\lambda$}

They are given by equalities $k=\sum_{1}^{n} L_{i}$ and

$$
\lambda=\frac{5^{2}}{2}(k-h)+w m+\frac{1}{2}\left[18.5 \cdot s_{\sqrt{37}}+\left(10+\frac{1}{8}\right) \cdot s_{4.5}+\left(15+\frac{1}{8}\right) \cdot s_{5.5}\right]
$$

where $w$ is defined as in Lemma 4, $s_{u}$ is the total number of segments of length $u$ in all $\Gamma_{i}$ 's for $u \in\{\sqrt{37}, 4.5,5.5\}$, and $h=m+\frac{1}{2}\left(s_{\sqrt{37}}+s_{4.5}+s_{5.5}\right)$.

It is easy to see that every $\{2,3\}$-clustering $\pi$ of $X$ into $k$ clusters must contain exactly $m$ triangles. Indeed, if $n_{T}$ and $n_{S}$ denote, respectively, the number of triangles and the number of segments of $\pi$, then $|X|=2 n_{S}+3 n_{T}=2 k+m$ and $n_{S}+n_{T}=k$, which yields $n_{T}=m$ and $n_{S}=k-m$. Recall that all triangles in $X$ have weight at least $w$. Moreover, by construction, $\pi$ may include at most $s_{u} / 2$ many segments of length $u$ for each $u \in\{\sqrt{37}, 4.5,5.5\}$ and the remaining $k-h$ cannot have length smaller than 5 . This implies $W_{2}(\pi) \geq \lambda$.

Now, to complete the reduction we verify that $\Phi$ is satisfiable if and only if there exists a $\{2,3\}$-clustering of $X$ of weight at most $\lambda$, consisting of $k$ clusters. Suppose $\Phi$ is satisfiable and consider a satisfying assignment. For each variable $v_{i}$, choose clustering $\pi_{2}(i)$ or $\pi_{1}(i)$ according whether its value is 0 or 1 , respectively. Since the assignment makes all clauses true, each point $z_{j}$ can be clustered together with the touched segment in $\Gamma_{i}$, for a variable $v_{i}$ satisfying clause $c_{j}$. By the parity condition, such a touched segment belongs to the chosen clustering 
(either $\pi_{2}(i)$ or $\pi_{1}(i)$ ). Thus, we obtain $m$ triangles of weight $w$. The other points in each $\Gamma_{i}$ can be clustered as in $\pi_{2}(i)$ or $\pi_{1}(i)$ according to the previous choice. This yields a $\{2,3\}$-clustering of $X$ of weight $\lambda$ having $k$ clusters.

Vice-versa, if there exists a $\{2,3\}$-clustering $\pi$ of $X$ with $k$ clusters and weight $\lambda$, then such a clustering must contain $m$ triangles of weight $w$. The only way to obtain these triangles is to include each point $z_{j}$ into a touched segment $\left\{x_{i t}, x_{i(t+1)}\right\}$. By the parity condition this defines an assignment of values to all variables that makes true each clause of $\Phi$.

Theorem 5. Assuming $\ell_{2}$-norm, the $\{2,3\}-R S C-2$ problem is strongly NP-hard and it does not admit an FPTAS unless $P=N P$. As a consequence, the same holds in general for $R S C$-2 problem.

Proof. The NP-hardness follows from the discussion above. The problem is also strongly NP-hard since the value of all integers in instances $(X, k, \lambda)$ obtained by the reduction is polynomially bounded w.r.t. $n=|X|$. Moreover, the objective function to minimize is polynomially bounded with respect to the unary size of the instance, and hence, by a classical result [23, Sec. 8.3], the same problem does not admit an FPTAS unless $P=N P$.

\section{Conclusions}

In this work, we have studied the clustering problem with relaxed size constraints in dimension 1 and 2 (RSC- 1 and RSC-2). First, we have shown a polynomialtime algorithm for RSC-1 in the case of $\ell_{1}$ and $\ell_{2}$-norm. A natural question is whether similar algorithm exists also for $\ell_{p}$-norm with integer $p>2$. We recall that the clustering in dimension 1 is motivated by bioinformatics applications as illustrated in 4 .

Our second result states that $\mathcal{M}$-RSC-2 problem is strongly NP-hard when $\mathcal{M}=\{2,3\}$. Note that with $\mathcal{M}=\{2\}$ the problem reduces to finding a perfect matching of minimum cost in a weighted complete graph, and hence it is solvable in $O\left(n^{3}\right)$ time (even in arbitrary dimension) assuming any $\ell_{p}$-norm, by using classical algorithms [18. The same occurs when $\mathcal{M}=\{1,2\}$ since this is reducible to finding the minimum cost matching of given cardinality in a weighted graph, which is known to be solvable in polynomial time (see for instance [20, sec. 3.1.1]). Hence, a natural problem is to determine the sets $\mathcal{M}$ for which the $\mathcal{M}$-RSC-2 problem is NP-hard.

Finally, we conjecture that $\{2,3\}$-RSC-2 remains NP-hard also in case of $\ell_{1}$-norm, by a suitable extension of the proof above.

Acknowledgments We thank an anonymous referee for his/her useful comments on other problems related to clustering with relaxed size constraints.

\section{References}

1. D. Aloise, A. Deshpande, P. Hansen, and P. Popat. NP-hardness of Euclidean sum-of-squares clustering. Machine Learning, 75:245-249, 2009. 
2. S. Basu, I. Davidson, and K. Wagstaff. Constrained Clustering: Advances in Algorithms, Theory, and Applications. Chapman and Hall/CRC, 2008.

3. A. Bertoni, M. Goldwurm, J. Lin, and F. Saccà. Size Constrained Distance Clustering: Separation Properties and Some Complexity Results. Fundamenta Informaticae, 115(1):125-139, 2012.

4. A. Bertoni, M. Rè, F. Saccà, and G. Valentini. Identification of promoter regions in genomic sequences by 1-dimensional constraint clustering. In Neural Nets WIRN11, pages 162-169, 2011.

5. C. Bishop. Pattern Recognition and Machine Learning. Springer, 2006.

6. P. S. Bradley, K. P. Bennett, and A. Demiriz. Constrained K-Means Clustering. Technical Report MSR-TR-2000-65, Miscrosoft Research Publication, May 2000.

7. S. Dasgupta. The hardness of $k$-means clustering. Technical Report CS2007-0890, Dpt. of Computer Science and Engineering, Univ. of California, San Diego, 2007.

8. W. D. Fisher. On grouping for maximum homogeneity. Journal of the American Statistical Association, 53(284):789-798, 1958.

9. U. Fößmeier, G. Kant, and M. Kaufmann. 2-Visibility drawings of planar graphs. In Graph Drawing (Proc. GD '96), volume 1190 of LNCS, pages 155-168, 1997.

10. S. Hasegawa, H. Imai, M. Inaba, and N. Katoh. Efficient algorithms for variancebased $k$-clustering. In Proceedings of Pacific Graphics '93, pages 75-89, 1993.

11. D. E. Knuth and A. Raghunathan. The problem of compatible representatives. SIAM J. Discrete Math., 5(3):422-427, 1992.

12. D. Lichtenstein. Planar formulae and their uses. SIAM J. Comput., 11(2):329-343, 1982.

13. J. Lin, A. Bertoni, and M. Goldwurm. Exact algorithms for size constrained 2-clustering in the plane. Theoretical Computer Science, 2015. doi:10.1016/j.tcs.2015.10.005. In Press.

14. S. Lloyd. Least squares quantization in PCM. IEEE Transactions on Information Theory, 28(2):129-137, 1982.

15. J. B. MacQueen. Some method for the classification and analysis of multivariate observations. In Proc. 5th Berkeley Symp. on Math. Struct., pages 281-297, 1967.

16. M. Mahajan, P. Nimbhorkar, and K. Varadarajan. The planar k-means problem is NP-hard. Theoretical Computer Science, 442:13-21, 2012.

17. W. Mulzer and G. Rote. Minimum-weight triangulation is NP-hard. J. ACM, 55(2), 2008.

18. C. Papadimitriou and K. Steiglitz. Combinatorial Optimization: Algorithms and Complexity. Dover, 1998.

19. M. R. Rao. Cluster Analysis and Mathematical Programming. Journal of the American Statistical Association, 66(335):622-626, 1971.

20. R. Stephan. Cardinality constrained combinatorial optimization: Complexity and polyhedra. Discrete Optimization, 7(3):99 - 113, 2010.

21. A. Tung, J. Han, L. Lakshmanan, and R. Ng. Constraint-based clustering in large databases. In Database Theory ICDT 2001, volume 1973 of LNCS, pages 405-419.

22. A. Vattani. $k$-means Requires Exponentially Many Iterations Even in the Plane. Discrete $\&$ Computational Geometry, 45(4):596-616, 2011.

23. V. Vazirani. Approximation Algorithms. Springer, 2001.

24. H. Vinod. Integer programming and the theory of grouping. Journal of the American Statistical Association, 64(326):506 - 519, 1969.

25. K. Wagstaff and C. Cardie. Clustering with instance-level constraints. In Proc. 17th Intl. Conf. on Machine Learning, pages 1103-1110, 2000.

26. S. Zhu, D. Wang, and T. Li. Data clustering with size constraints. KnowledgeBased Systems, 23(8):883-889, 2010. 